\title{
Heurs et malheurs de deux saints nationaux : Patrick et Brigitte dans la tourmente politique (1850-2014)
}

\section{Catherine Maignant}

\section{(2) OpenEdition}

\section{Journals}

\section{Édition électronique}

URL : http://journals.openedition.org/etudesirlandaises/4498

DOI : 10.4000/etudesirlandaises.4498

ISSN : 2259-8863

\section{Éditeur}

Presses universitaires de Rennes

Édition imprimée

Date de publication : 30 juin 2015

Pagination : 165-183

ISBN : 978-2-7535-4082-8

ISSN : 0183-973X

\section{Référence électronique}

Catherine Maignant, « Heurs et malheurs de deux saints nationaux : Patrick et Brigitte dans la tourmente politique (1850-2014) », Études irlandaises [En ligne], 40-1 | 2015, mis en ligne le 30 juin 2017, consulté le 20 avril 2019. URL : http://journals.openedition.org/etudesirlandaises/4498 ; DOI 10.4000/etudesirlandaises.4498 


\title{
Heurs et malheurs de deux saints nationaux : Patrick et Brigitte dans la tourmente politique (1850-2014)
}

\author{
Catherine Maignant \\ Université de Lille 3
}

\section{Résumé}

Les saints irlandais, particulièrement Patrick et Brigitte, ont été mis à contribution dès le $\mathrm{XIX}^{\mathrm{e}}$ siècle pour construire la nation catholique. La vogue de l'hagiographie, et la manière de rédiger les vies de saints au cours des dernières décennies du siècle, en portent témoignage. Au moment de l'indépendance et dans les années qui ont suivi, le culte organisé de saints privilégiés, ainsi que son étatisation ont assigné à ces personnages une fonction nationale à visée identitaire et politique. Après avoir retracé les grandes étapes de l'instrumentalisation des grands saints nationaux, l'article s'interroge sur l'effet de la prise de distance de l'État vis-à-vis de l'Église catholique sur leur culte.

Mots clés : Église catholique d'Irlande, société et religion, débat et sphère publics, État (République d'Irlande), identité - nationale.

\section{Abstract}

From the nineteenth century, Irish saints -Patrick and Brigit in particular-were made to contribute to the construction of the Catholic nation. The interest in hagiography and the way saints' lives were written in the final decades of the nineteenth century bear witness to this phenomenon. In the decades that followed the creation of the Irish Free State, the worship of privileged saints was organized at state level and a national mission was somehow ascribed to these figures, who came to play a key role in the identity formation process. After retracing the major steps in the instrumentalization of the great national saints, this article analyses the contemporary developments of the question, following the evolution of Church-State relationships in Ireland.

Keywords: Irish Catholic Church, society and religion, Public debates, Irish State (Republic of Ireland), identity - national

Des saints d'Irlande, Patrick et Brigitte sont ceux que la mémoire collective a investis au cours des âges des valeurs les plus fortes. L'histoire les a portés au pinacle, elle leur a accordé une succession de vies lumineuses en leur confiant des charges aussi diverses que contradictoires. Champions de causes parfois communes, parfois antagonistes, Patrick et Brigitte ont connu une destinée exceptionnelle qui ne paraît pas aujourd'hui avoir encore atteint son terme. Si leur origine 
se perd dans les brumes du haut Moyen Âge, ils sont, l'un et l'autre, associés à l'identité irlandaise que le $\mathrm{XIX}^{\mathrm{e}}$ siècle a voulue immémoriale. Tous deux ont été convoqués pour construire la nation catholique et accompagner l'avènement de l'État-nation. À la naissance de l'État Libre, ils ont été figés dans le rôle de saints nationaux primordiaux, gravés dans l'espace public par la statuaire et l'architecture ecclésiastiques, littéralement pétrifiés dans le sanctuaire érigé à la gloire de la sainte Irlande. Leur culte et leurs commémorations ont été soigneusement codifiés, soumis à la toute puissante Église de l'ère de Valera.

Leur destin ultérieur a suivi l'évolution de l'État irlandais. Les transformations successives du mode de célébration de la fête nationale, le jour de la Saint-Patrick, sont symboliques de la prise progressive de distance de l'État vis-à-vis de l'Église. À l'inverse, le maintien, à Croagh Patrick et Lough Derg, de pèlerinages pénitentiels toujours ancrés dans la tradition catholique la plus rigide signale à l'observateur l'écartèlement véritable dont le saint fait l'objet dans la symbolique idéologique. Brigitte, pour sa part, a clairement entamé une nouvelle carrière, qui la porte désormais à la pointe du renouveau religieux en Irlande et bien au-delà des frontières de la République. Les manipulations récentes des deux saints nationaux suggèrent qu'ils sont toujours porteurs de sens dans une Irlande en quête de renouveau identitaire et religieux. Leur étrange aventure, vibrante d'une vie mythique réinterprétée à chaque tournant de l'histoire de l'Irlande fait écho aux angoisses et aspirations de temps divers. Ce sont aujourd'hui deux visions du monde adverses qu'ils sont appelés à porter.

Après avoir retracé les grandes étapes de l'instrumentalisation des deux personnages, le présent article observera au prisme de leur culte et de leurs commémorations l'évolution religieuse de l'Irlande, le rapport entre l'État et l'Église, et l'inscription de la République d'Irlande dans le monde. Les différentes formes de vénération dont font encore l'objet Patrick et Brigitte résument et subsument les questionnements clés de la société irlandaise contemporaine, ses crispations, ses tâtonnements, ses résistances au conformisme comme à l'anticonformisme, ses ouvertures vers l'avenir comme ses attachements à l'héritage du passé. De l'Irlande archaïque au monde ultramoderne globalisé, de l'histoire aux mythes et au folklore, les deux saints nationaux ont été façonnés pour incarner les idéaux d'un peuple. Ils contribuent aujourd'hui à la naissance délicate de l'Irlande post-catholique.

\section{Les origines}

Si l'on en croit la tradition hagiographique, Saint Patrick et Sainte Brigitte sont à peu près contemporains. Tous deux compteraient en effet parmi les premiers évangélisateurs de l'Irlande au $\mathrm{v}^{\mathrm{e}}$ siècle. C'est toutefois au $\mathrm{VII}^{\mathrm{e}}$ siècle qu'ils 
sortent de l'obscurité des premiers âges pour acquérir une signification politique. Les vies de Patrick par Muirchú et Tirechán ont en effet pour ambition de faire de leur héros l'apôtre de l'Irlande qu'il n'a sans doute jamais été en réalité, afin de légitimer la revendication d'Armagh à la primauté épiscopale. Dans cette lutte politique, Patrick se trouve confronté à Brigitte au nom de qui Kildare recherche le même privilège par le biais d'une vie rédigée par Cogitosus, semble-t-il vers le milieu du siècle. Armagh répond à Kildare et emporte la victoire : Patrick sera le principal saint d'Irlande et Brigitte viendra en second.

La réussite du camp patricien n'est pas anodine au vu de l'histoire ultérieure de l'Église puisque la querelle s'inscrit dans un contexte de lutte entre la mouvance " romaine ", favorable à la mise en conformité totale avec Rome et la tendance " irlandaise ", soucieuse de préserver les particularités de l'Église celtique. Il fait peu de doutes que les hagiographes de Patrick, saint étranger, étaient des Romains convaincus, désireux d'imposer un système épiscopal fort aux dépens du modèle monastique prisé par l'Église des premiers siècles. Bien que Cogitosus fasse de Brigitte un évêque (ordonné par erreur), son héroïne est ancrée dans la tradition irlandaise, qu'elle incarne tout particulièrement dans sa fonction d'abbesse du monastère double de Kildare, érigé à proximité d'un temple dédié à la grande déesse païenne Brigitte, avec qui il est facile de la confondre.

Il est indiscutable, en tous les cas, que les deux saints avaient acquis une position dominante au $\mathrm{VII}^{\mathrm{e}}$ siècle, ce qui est confirmé notamment par l'œuvre supposée de l'évêque Ultán d'Árd-mBrecáin, connu pour avoir été l'inspirateur de Tirechán, et dont une tradition ancienne veut qu'il ait aussi écrit une vie de Brigitte. On lui attribue en outre la paternité de l'hymne le plus ancien qui nous soit parvenu en l'honneur de la sainte, qui fait d'elle la "mère de Jésus " ». Au $\mathrm{x}^{\mathrm{e}}$ siècle, elle allait devenir " La Marie des Gaëls » dans un panégyrique extrait du Leabhar Breac, qui précise : "Le père de cette vierge sainte était le Père des Cieux, son fils était Jésus Christ ${ }^{2}$. " Unis par la tradition haut-médiévale, les deux saints suggéraient symboliquement la fusion originale des cultures sous l'autorité patriarcale ultime de l'Église de Rome. Brigitte la déesse mère revêtait les oripeaux de la sainte et acceptait la tradition épiscopale tandis que Patrick, apôtre de la conformité romaine, remplaçait dans les légendes populaires un Dieu Lugh pourvoyeur d'abondance, et protecteur des hommes.

Alors que son homonyme, la déesse païenne, était célébrée le $1^{\text {er }}$ février, date de la fête celtique d'Imbolc, la sainte allait être commémorée le 2, fête de la purification de la Vierge en souvenir de sa présence supposée auprès de Marie à cette

1. Hymne dit d'Ultán, éd. Whitley Stokes, Goidelica, Londres, Trübner \& Co, 1872, p. 133-137.

2. Ed. James Todd, The Book of Hymns of the Ancient Church of Ireland, Dublin, Irish Archaeological and Celtic Society, 1855, Vol. 1, p. 65. 
occasion $^{3}$. La proximité des dates et la similarité des attributions des deux Brigitte allaient entretenir entre elles une confusion d'autant plus commode que la mort de la sainte serait intervenue un $1^{\text {er }}$ février. Patrick, pour sa part, était commémoré le jour anniversaire de son décès, fixé par la tradition au 17 mars. Le Livre d'Armagh, daté du Ix siècle, précise déjà que la Fête de "l'endormissement de Saint Patrick » devait être célébrée à cette date dans tous les monastères et églises ${ }^{4}$.

Lassociation entre Patrick et Brigitte allait rester suffisamment prégnante dans la mémoire collective médiévale pour que leurs corps soient mystérieusement retrouvés enterrés ensemble à Downpatrick dans les années 1180, ainsi que l'atteste Giraud de Cambrie, le célèbre chroniqueur anglo-normand du XII ${ }^{e}$ siècle ${ }^{5}$. Cet auteur confirme par la même occasion l'importance de Kildare dont il dit que « la glorieuse Brigitte l'a rendu célèbre ». Il évoque le feu que « les religieuses et saintes femmes » entretenaient si bien que, "depuis le temps de la vierge sainte jusqu'à maintenant, il n’a jamais été éteint ». Le feu fut finalement étouffé et l'abbaye dissoute en 1540 sur l'ordre de George Brown, archevêque de Dublin, dans le contexte de la Réforme. Bien que la sainte soit demeurée discrètement présente dans la tradition anglo-irlandaise anglicane, la grande tradition de Sainte Brigitte ne fut ensuite ravivée qu'au $\mathrm{XIX}^{\mathrm{e}}$ siècle dans des circonstances radicalement différentes.

Le XII ${ }^{\mathrm{e}}$ siècle mit à l'honneur Patrick tout autant que Brigitte, notamment par le biais du Tractatus de Purgatorio Sancti Patricii rédigé par Henri de Saltrey, un moine cistercien de l'abbaye de Saltrey et la célèbre Vita Patricii de Jocelyn de Furness, l'un et l'autre contemporains du texte de Giraud de Cambrie. Les trois auteurs évoquent un Purgatoire de saint Patrick, qu'ils situent dans des endroits différents, tout en portant témoignage du lien ancien entre Patrick, Lough Derg et Croagh Patrick, de la perpétuation du souvenir de ce lien jusqu'au XII ${ }^{\mathrm{e}}$ siècle et de la volonté des nouveaux arrivants normands de se couler dans la culture locale et glorifiant le saint apôtre. Patrick ne devait pas, en revanche, connaitre la relative éclipse dont Brigitte fit l'expérience au moment de la Réforme, bien au contraire, et c'est là un point de divergence aussi fondamental que durable entre les deux saints.

St Patrick, en effet, fut assimilé et glorifié par la tradition de l'Église réformée. Écrivant sa vie de Patrick en 1864, James Todd note : «Alors que l'Église irlandaise des origines [...] se fondit dans l'Église du Pale anglais et adopta la Réforme, elle perdit dans une large mesure son emprise sur les descendants des tribus indigènes, une perte que l'on peut attribuer au rejet ancien et profond de

3. Alexander Carmichael, Carmina Gadelica, 1900, Vol. 1, p. 169, [http://sacred-texts.com/neu/celt/cgl/index.htm], Consulté le 29 septembre 2014.

4. Noté par Mike Cronin et Daryl Adair, The Wearing of the Green, A History of St Patrick's Day, Oxon et New York, Routledge, 2002, p. xxix.

5. Saint Columba est également enterré avec eux. Gerald of Wales, The History and Topography of Ireland, c. 1185, éd. John O’Meara, Harmondsworth, Penguin Books (1951) 1982, p. 105. 
l'Angleterre ${ }^{6}$. " Cette annexion de Patrick à la tradition anglicane d'Irlande, qui causa bien des remous dans l'opinion catholique, nous le verrons, trouve un écho dans le fait que Patrick fut de tout temps honoré par la communauté anglo-irlandaise qui, à l'instar des Anglo-normands, souhaitait de cette manière s'intégrer au cadre religieux et culturel de l'île conquise. En 1607, la fête de Patrick fut ajoutée au calendrier légal de l'Irlande sous domination anglaise. Dans le même temps, néanmoins, Luke Wadding, érudit franciscain originaire de Waterford, suggérait d'ajouter le 17 mars à la liste des fêtes reconnues par l'Église catholique. Le Vatican soutint le culte de Patrick, intégré vers la même époque à la cohorte des saints catholiques bien qu'il n'ait jamais été canonisé. Cette double affiliation permit à Patrick de passer les siècles sans être inquiété et de se placer à l'origine d'une célébration d'autant plus consensuelle que le saint se vit élevé au statut de saint patron d'Irlande dans le courant du XVII ${ }^{e}$ siècle, afin, peut-être, qu'il soit possible de célébrer les racines chrétiennes communes de l'île.

\section{L'avènement de deux saints nationaux}

C'est au $\mathrm{XIX}^{\mathrm{e}}$ siècle que les destinées de Brigitte et Patrick se trouvèrent à nouveau réunies, moyennant quelques accommodements avec l'histoire et les mythes hérités des temps anciens. La première référence à l'abbesse à cette époque concerne l'établissement de l'ordre de Sainte Brigitte (Brigidine Order) par l'évêque de Kildare et Leighlin, Daniel Delany, le $1^{\text {er }}$ février 1807. Il s'agissait dans son esprit, non de fonder une nouvelle congrégation, mais de recréer l'ordre de Sainte Brigitte de Kildare, dont il était persuadé qu'il avait existé du $v^{\mathrm{e}}$ au $\mathrm{XVI}^{\mathrm{e}}$ siècles. Le nouveau couvent fut néanmoins établi à Tullow ${ }^{7}$, non à Kildare et aucun feu sacré ne fut allumé dans l'enceinte. Suite à l'abrogation des lois pénales, qui avaient privé les catholiques du droit à l'instruction, l'évêque ambitionnait simplement d'instituer une école du dimanche catholique dédiée à l'éducation des filles. La seule référence à la tradition fut la plantation, dans le parc de l'établissement, d'un jeune chêne ${ }^{8}$ que Daniel Delany fit spécialement apporter de Kildare.

La sainte devait ensuite se voir associée à la renaissance culturelle promue par les nationalistes en quête d'icônes pour la nation catholique. Elle fut également projetée sur le devant de la scène au moment de la réforme catholique de la deuxième moitié du siècle, encore souvent appelée "révolution dévotionnelle ", dont elle devint une figure populaire clé. Dans le contexte de la politique romaine visant à moraliser et civiliser le monde catholique et y asseoir l'autorité centralisa-

\footnotetext{
6. James Henthorn Todd, St Patrick Apostle of Ireland: A Memoir of his Life and Mission, Dublin, Hodges, Smith \& Co, 1864, p. v.

7. Tullow se situe à une soixantaine de kilomètres de Kildare.

8. Cill dara signifie l'église des chênes.
} 
trice du Vatican, la sainte irlandaise fut refaçonnée de manière à pouvoir servir de modèle aux Irlandaises. En 1854, le pape Pie IX accorda une licence spéciale autorisant l'Irlande catholique à célébrer la fête de Sainte Brigitte pendant la semaine faisant suite au $1^{\text {er }}$ février. Si les traditions folkloriques agraires issues de formes abâtardies de célébrations païennes survécurent, l'accent fut essentiellement placé sur les rituels catholiques.

Les érudits commencèrent à la même époque à s'intéresser à cette héritière de la double tradition gaélique et chrétienne de l'âge d'or irlandais. Plusieurs vies de Brigitte furent aussi publiées à cette période, dont l'une rédigée en 1861 par un prêtre anonyme qui, dans son introduction, affirme son désir " de faire mieux connaître aux Catholiques d'Irlande l'histoire et les vertus ${ }^{9}$ " de la sainte, un signe qu'elles étaient peut-être largement oubliées des lecteurs moyens. Quelques années plus tard, O'Hanlon exprima sa conviction de l'importance particulière de Brigitte en acceptant d'écrire d'elle une Vie spécifique distincte de son projet d'ouvrage intitulé Lives of Irish Saints, un privilège que seuls quelques saints, dont Patrick, devaient avoir par ailleurs. L'auteur de la Vie de 1861 voyait en Brigitte la sainte patronne particulière des diocèses de Kildare et Leighlin. O'Hanlon ajoute qu'elle est " la sainte patronne générale d'Irlande ", un statut que l'évêque Delany lui reconnaissait déjà. Dans sa préface, il remarque que les religieux dont l'institution a été placée sous son patronage ont besoin "d'en savoir plus sur ses vertus et miracles supérieurs " tandis que le grand public trouvera utile de découvrir les détails de la vie de Brigitte, qui « leur permettront d'accroître (leur) amour et leur vénération " pour la sainte. Il ajoute en outre que Brigitte doit être considérée comme un modèle pour les femmes qui, par milliers, ont reçu son nom par le baptême ou la confirmation. "Son exemple lumineux a amené de nombreuses Irlandaises au statut de sainteté sur terre et à une récompense éternelle au paradis ", écrit-i $1^{10}$.

La réécriture régulière de la vie de Brigitte à cette époque a une valeur commémorative indiscutable en ce qu'elle réintègre la sainte délaissée dans la mémoire collective centrale. Mais elle permet également de justifier les initiatives de l'Église contemporaine et contribue à l'élaboration de l'avenir en proposant un exemple à suivre pour l'élément féminin de la société. Il n'y avait en 1800 que 122 religieuses en Irlande. Elles étaient 8000 en 1900, à la fois actives et contemplatives, comme Brigitte, ainsi que O'Hanlon le souligne. Son lien avec la maternité est aussi souligné, un signe fort pour les femmes qui ne souhaitaient pas rejoindre

9. The Life of Saint Bridget, "The Mary of Erin", and Special Patroness of the Dioceses of Kildare and Leighlin by an Irish priest, 1861, p. 4. [http://books.google.co.uk/boo,ks?id=AJZjAAAAMAAJóprintsec=frontcover $\# v=$ onepaget $q$ boff=]. Consulté le 29 septembre 2014.

10. Rev John O'Hanlon, Life of St Brigid, 1877, p. vIII-IX, [https://archive. org/details/lifeofstbrigidviO0ohanuoft]. Consulté le 29 septembre 2014. 
une communauté et se voyaient orientées vers le mariage, la vie domestique et la reproduction.

Si le renouveau du culte de Patrick sous l'égide du nationalisme s'inscrit sensiblement dans la même temporalité que celui de Brigitte, son histoire est plus complexe. Son statut d'apôtre de l'Irlande et de saint patron principal de l'île ainsi que son lourd passé d'association avec l'Église anglicane compliquent en effet singulièrement les enjeux. Les phases et les manifestations du renouveau d'intérêt pour la personnalité de Patrick sont ainsi comparables à ce qui vient d'être dit à propos de Brigitte, mais les stratégies mises en œuvre s'insèrent dans trois logiques distinctes.

En premier lieu vient la tentative protestante de maintenir son emprise sur Patrick et les efforts catholiques pour le récupérer. Nous avons évoqué plus haut le cas de James Todd qui, outre l'annexion de la première Église irlandaise à l'Église anglicane d'Irlande rejette tout lien du saint avec Rome : selon lui, il n'a pas été envoyé en Irlande par le pape Célestin, il n’a jamais apporté de reliques de Rome; il n'a jamais été accueilli ni au Vatican ni dans un monastère gaulois; il n'a jamais été ermite augustinien; de plus, la tradition du Purgatoire de Patrick est tardive. Todd n'hésite pas à parler de " fables ", d' "imposture » et de " superstitions » véhiculées par les vies tardives de Patrick, résultat des agissements d'auteurs " peu scrupuleux ». Ce faisant, écrit-il, il fait œuvre d'historien et n'est en rien guidé par les préjugés anticatholiques puisque " la Rome du v siècle n'était pas coupable des abus qui ont rendu la Réforme nécessaire ${ }^{11}$ ". Les protestants toutefois ne parlent pas d'une seule voix et, comme chacun sait, toutes les obédiences n'ont pas le respect des anglicans envers les saints en général. Dans le cas de Patrick cependant, tous rejettent le lien avec Rome et l'élément miraculeux des vies tardives que la tradition catholique accepte sans aucun recul critique. Certains, à l'instar de Ledwich, vont d'ailleurs jusqu'à nier l'existence de Patrick, fruit de l'imagination trop fertile des «bardes toujours prêts à promouvoir les rêves et désirs stupides du peuple $^{12} »$.

La réponse catholique est double. Se mêle en effet au débat le fait que les protestants se sont beaucoup plus intéressés à Patrick que les catholiques, et que nombre d'entre eux, intellectuels et historiens, se réclament de la science historique naissante pour faire le tri dans le fatras des traditions relatives au saint patron de l'Irlande. Les contradicteurs catholiques s'attaquent donc à une double tâche : défendre la tradition complète de l'Église catholique à propos de Patrick et lutter contre la démarche scientifique qui contamine de la même manière érudits protestants et catholiques. Dans le premier ouvrage rassemblant sans distinction toutes les traditions relatives à Patrick, The Life of St Patrick Apostle of Ireland,

11. James Henthorn Todd, op. cit., p. vi.

12. Cité par William Bullen Morris, Ireland and St Patrick, Londres et Dublin, Gill \& Sons, 2édition, 1878 , p. 1. 
publié par Mary Cusack en 1871, l'auteur se lance dans une violente diatribe contre ceux qui se laissent guider sur le chemin de la connaissance par «la lampe de la science " susceptible de précipiter ses adeptes dans "les sables mouvants de l'erreur ". Seule, à ses yeux, "la lampe de la foi " permet en effet d'acquérir le vrai savoir, et c'est à un théologien que Mme Cusack s'adressera pour vérifier la validité de ses dires puisque Dieu, "la source de toute connaissance ", "doit aussi être la source de toute science ${ }^{13}$ ». De même William Bullen Morris répudie-t-il, " au nom du sens commun et de la science sacrée ", "l'usurpation dont se rend coupable cette "histoire scientifique" qui aspire à disséquer et séculariser une biographie si surnaturelle ${ }^{14}$ ".

Pour l'un comme pour l'autre, ainsi que pour tous les auteurs de Vies qui souhaitent écrire pour l'édification d'un public catholique, il convient de lutter contre ces tendances néfastes et rétablir la vérité, celle qui dit les miracles de Patrick auxquels on croit " parce que ces choses sont simplement ce qu'un catholique s'attend à trouver dans la vie d'un saint ${ }^{15}$ ". "L'histoire de Saint Patrick constitue un test de foi ", note William Bullen Morris dans la quatrième édition de son ouvrage, publiée en $1891^{16}$.

Que la rédaction de plusieurs vies catholiques de ce type soit liée à la volonté d'asseoir la puissance de Rome au sein de la nation catholique est patent. L'action de Pie IX est évoquée ici ou là, son "Église infaillible ${ }^{17}$ » est le cadre dans lequel se situe la propagande patricienne, et Patrick est un agent de Rome, fondateur d'une Église orthodoxe qui a essaimé en Europe et a eu un rôle primordial dans la conversion de l'Angleterre même. ${ }^{18}$ En 1905, Bury poussera cette conviction à l'extrême. Selon lui, l'évangélisation de l'Irlande est " une annexe de l'histoire de l'Empire romain ${ }^{19}$ " et l'une des contributions principales de Patrick a été de " mettre l'Irlande en contact avec l'Église de l'Empire et de l'intégrer officiellement dans la chrétienté universelle ${ }^{20} »$. Il convient donc de respecter la volonté de Patrick, "porteur de l'idée romaine " à une époque où " le pape n'était pas encore devenu le Caesar Augustus qu'il est aujourd'hui ${ }^{21}$ ». Le message du début du $\mathrm{xx}^{\mathrm{e}}$ siècle est limpide : il faut soutenir une Église ultramontaine soumise à Rome et respecter en tout les directives papales.

13. Mary Cusack, The Life of St Patrick Apostle of Ireland, Londres, Longmans, Green \& Co, 1871, p. 7-8.

14. William Bullen Morris, op. cit., p. 33.

15. Ibid., p. 17.

16. William Bullen Morris, Ireland and St Patrick, Londres et Dublin, Gill \& Sons, 1891, p. 35.

17. Mary Cusack, op. cit., p. 9. Pie IX a déclaré l'infaillibilité papale en 1870 en la limitant aux déclarations ex cathedra. Notons que, un an après la proclamation, l'Église tout entière est devenue infaillible à tout moment dans la perception de Mary Cusack.

18. William Bullen Morris op. cit. 1891, p. 26.

19. J. B. Bury, The Life of St Patrick and his Place in History, Londres, Macmillan \& Co, 1905, p. i.

20. Ibid., p. 212.

21. Ibid., p. 221. 
Il est manifeste que le sentiment nationaliste accompagne l'initiative visant au renouveau du culte de Patrick. Mary Cusack souhaite produire un livre magnifique "à la fois au nom de notre religion et de notre nationalité ". Elle souhaite par ailleurs « la régénération de l'Irlande " grâce au clergé irlandais qu'elle soutient en transmettant le savoir, car " connaissance est synonyme de pouvoir » et " une nation puissante ne peut en aucun cas être une nation piétinée ${ }^{22}$ " Néanmoins, le texte de Bury met en garde le lecteur contre l'Église celtique mise en avant par Todd, qu'il ne souhaite pas défendre dans la venue à maturité d'une nation véritablement catholique suivant le modèle de Patrick. En effet, écrit-il, « les tendances qui se sont affirmées après la mort de Patrick peuvent être en partie comprises comme relapses ${ }^{23}$ ». L'Église monastique du Christianisme celtique et ses spécificités culturelles sont donc dévalorisées et traitées avec méfiance alors que l'institution romaine et son message à valeur universelle sont envisagés comme le modèle ultime à défendre, le seul qui rompe l'isolement et les particularismes de l'Irlande pour intégrer l'île dans une structure internationale garante du respect de la vérité divine.

\section{圈 Du culte à la commémoration : l'institutionnalisation du culte des deux saints nationaux}

Le lien grandissant entre l'Église et l'idéal de la nation, puis de l'État-nation allait aboutir à l'institutionnalisation de la vénération des deux saints nationaux. Le poids de l'Église dans ce mouvement fut tout à fait considérable. De grandes commémorations collectives furent organisées pour donner sa pleine mesure et sa pleine visibilité à l'autorité croissante du clergé, fondée sur sa fonction médiatrice entre l'humain et le divin. À une époque où le sens du péché, de la honte et de la culpabilité étaient renforcés dans le monde catholique, l'Église réactiva la pratique des pèlerinages pénitentiels afin d'inciter les fidèles à rechercher l'intercession des saints et le pardon divin. Patrick et Brigitte furent, l'un et l'autre, mis à contribution dans ce cadre particulier.

Le Dr Healy, archevêque de Tuam, lui aussi auteur d'une Vie de Saint Patrick en $1905^{24}$ relance ainsi au début du $\mathrm{xx}^{\mathrm{e}}$ siècle le pèlerinage de Croagh Patrick, qui était tombé en désuétude au moment de la Grande Famine. J. Stephens et M. Clarke, deux prêtres conscients de la nécessité d'associer Patrick à la renaissance de la culture irlandaise avaient bien essayé d'engager le mouvement en faisant ériger une chapelle temporaire en 1882 mais la tentative n'avait pas

22. Mary Cusack, op. cit., p. 10.

23. J. B. Bury, op. cit., p. 215.

24. John Healy, The Life and Writings of Saint Patrick, 1905, accessible en ligne à l'adresse suivant : [https://archive. org/details/lifewritingsofstOOhealrich]. Site consulté le 25 septembre 2014. 
été soutenue par l'archevêque de l'époque. Vingt ans plus tard, Healy est, au contraire, enthousiaste. Dès 1903, il soutient l'initiative du père McDonald de célébrer à nouveau la messe au sommet de la montagne et il fait affréter des trains pour que les pèlerins affluent. Puis il ordonne la construction de l'oratoire de saint Patrick en 1904 tout en maintenant son arrangement avec les compagnies de chemin de fer. Dix mille pèlerins feront le voyage pour l'inauguration du bâtiment en 1905. Aux yeux de l'archevêque, Croagh Patrick est le symbole de la foi inébranlable de l'Irlande et cette montagne sacrée devrait devenir " le lieu d'un pèlerinage national ", symbole du lien inexpugnable entre la foi catholique et la nation irlandaise, que rien n'avait jamais ébranlées ni ne détruirait jamais ${ }^{25}$.

Le lien entre pèlerinage et nation fut renforcé dans les années 1920 et 1930, à mesure que la puissance de l'Église catholique grandissait au sein de l'État. Il fut aussi inscrit dans le paysage par diverses réalisations architecturales visant à pérenniser l'autorité de l'institution. La construction de l'église de Station Island dans le Lough Derg fut engagée en 1925 et le bâtiment, « honoré du titre de basilique par le pape Pie $\mathrm{XI}^{26}$ ", fut inauguré en 1931. Il venait remplacer l'ancienne église Saint Patrick et s'ajouter à l'église Sainte Marie, érigée en 1870. Dans son ouvrage sur Lough Derg, dont sont connues deux éditions datées respectivement de 1928 et de 1934, J. E McKenna parle désormais d'un pèlerinage national ${ }^{27}$. Dès 1932, Eamon de Valera faisait par ailleurs le pèlerinage de Croagh Patrick à Reek Sunday, le dernier dimanche de juillet, célébration principale organisée sur la montagne, héritière de Lughnasad, fête des moissons du $1^{\text {er }}$ août en l'honneur du dieu Lugh à l'époque païenne.

Dans le même temps, Brigitte confirmait son statut de sainte nationale et son culte était délibérément ravivé par les autorités ecclésiastiques soutenues par l'État. Dans plusieurs de ses numéros, le Catholic Herald mentionne un certain père McCarroll qui, dans les années 20, aurait été " le champion de la renaissance du culte de Ste Brigitte ${ }^{28}$ " et aurait " accompli une tâche gigantesque en rendant (la sainte) à la nation ${ }^{29}$ ». En 1924, il fit en effet construire une nouvelle église sur le site d'une ancienne chapelle Sainte Brigitte, qu'il souhaitait dédier au sacré-Cœur ou à sainte Ita. Mais c'est le nom de Sainte Brigitte qui fut choisi par les autorités ecclésiastiques. Le bâtiment fut inauguré par l'archevêque de Dublin deux ans plus tard

25. Harry Hughes, Croagh Patrick - A Place of Pilgrimage, a Place of Beauty, Dublin, O’Brien Press, 1991, p. 46-47. 26. Eileen Good, Places Apart - Lough Derg, Dublin, Veritas, 2003, p. 18.

27. J.E. McKenna, Lough Derg, Ireland's National Pilgrimage, Dublin, Catholic Trust Society of Ireland, 1928 et 1934.

28. Catholic Herald, UK, 9 juillet 1937, [http://archive.catholicherald.co.uk/article/9th-july-1937/10/st-brigidsshrine], Consulté le 30 septembre 2014.

29. Catholic Herald, UK, 12 February 1937. [http:/larchive.catholicherald.co.uklarticle/12th-february-1937/11/irishletter]. Consulté le 30 septembre 2014. 
et le processus fut complet en 1928 lorsqu'un fragment du crâne de Brigitte y fut apporté du Portugal, où la tête de la sainte est officiellement conservée.

À la suite de ces événements, il fut décidé qu'une commémoration nationale en l'honneur de Brigitte serait organisée à Faughart où la sainte serait née. À l'initiative du Cardinal McRory un nouveau sanctuaire y fut érigé ainsi qu'un chemin de croix et une grotte sur le modèle de celle de Lourdes. Des reliques furent solennellement apportées et un pèlerinage national fut lancé. Le Taoiseach Eamon de Valera, plusieurs membres de son gouvernement et le maire de Dublin participèrent à sa première édition en 1934 et se joignirent aux dix à quinze mille participants.

Faughart remplaça donc Kildare comme site privilégié de commémoration. De plus, une date en juillet remplaça le $1^{\text {er }}$ (ou le 2) février. Ce déplacement spatial et temporel fut à l'évidence soigneusement réfléchi. La cathédrale Sainte Brigitte à Kildare qui, de manière significative, avait été restaurée à la fin du XIx ${ }^{e}$ siècle, appartenait à l'Église anglicane (Church of Ireland), d'où le choix d'un lieu différent. $\mathrm{Si}$, par ailleurs, d'un point de vue climatique, le mois de juillet était manifestement plus propice à un pèlerinage que février, le changement de date, comme l'absence de référence à Kildare, avaient également le mérite d'éliminer la déesse païenne du paysage. Dans sa Vie de Brigitte publiée en 1942, Aodh de Blacam niait en bloc le lien que les historiens de son époque établissaient entre la sainte et la déesse. Parlant de Kenney, il écrivait qu'il était surpris de voir un si bon historien "soutenir une théorie aussi peu orthodoxe $\mathrm{e}^{30}$ ". Histoire et mémoire sont deux choses différentes et les commémorations, loin d'inciter les participants à s'informer sur le passé, contribuent au contraire à façonner et éduquer la mémoire collective, comme le suggère Delumeau ${ }^{31}$. Il semble en réalité que la distanciation soit cruciale pour que le phénomène d'appropriation se produise. La dé-contextualisation est même un élément si essentiel que la fonction des commémorations semble être bien moins de faire revivre le passé que de le manipuler pour répondre aux besoins du présent et construire l'avenir.

Sensiblement à l'époque où Aodh de Blacam écrivait sa Vie de la sainte, la fête de Brigitte fit d'ailleurs l'objet d'une enquête de l'Irish Folklore Commission, qui mit en évidence la distance considérable entre les survivances folkloriques et la mise en scène spectaculaire retenue par l'Église pour fêter la sainte nationale.

30. Aodh de Blacam, «St Brigid, the Mary of the Gael » in The Saints of Ireland: The Life Stories of Ss Brigid and Columcille, 1942, chapter 2, [http://www.ewtn.com/library/MARY/BRIGID.htm/]. Consulté le 30 septembre 2014.

31. « Pourquoi ne pas le dire? Au moment où tant d'événements encombrent le présent, les commémorations se veulent à la fois pédagogiques et civiques en pointant du doigt des faits ou des personnages qui ont lancé l'humanité, et plus particulièrement celle d'Occident, dans la voie qui est la sienne aujourd'hui. „Célébrations nationales 2002, Préface de Jean Delumeau, [http://www.culture.gouv.fr/culturelactualites/celebrations2002/preface.htm]. Consulté le 30 septembre 2014. 
Des pratiques traditionnelles, l'État et l'Église choisirent de ne préserver que la visite aux puits guérisseurs et le tressage de croix de sainte Brigitte. Une croix de ce type fut même étonnamment choisie comme symbole du ministère de la Santé dans les années 40 et 50 et de RTÉ, la chaîne de télévision nationale, à partir de 1961. Dans un article consacré à ces étranges associations, le Design Research Group défend l'idée que ces symboles rassurants « des mythes sociétaux de l'État irlandais, religieux, traditionnels, domestiques » étaient utilisés pour " rendre la nouveauté familière afin de permettre à l'État de pénétrer dans la sphère domestique de la manière la moins conflictuelle possible ${ }^{32}$ ». Brigitte était apprivoisée, transformée en signifiant visuel inoffensif. Dès 1937, la sainte dépouillée de tout relent de paganisme et de protestantisme se voyait comparée par le Catholic Herald à un Saint Patrick purement catholique romain. L'un de ses numéros appelait de ses vœux une reconnaissance toujours plus grande : «Espérons que bientôt entrera dans les mœurs une célébration de la Sainte Brigitte comme jour férié religieux national comparable à la Saint Patrick ${ }^{33}$. "

Cette dernière fête mérite d'ailleurs que l'on s'y arrête quelques instants. Si l'on en croit le magistral ouvrage de Mike Cronin et Daryl Adair sur l'histoire de la Saint Patrick, l'association grandissante entre le religieux et le politique, essentiellement à partir de la fin du XIX ${ }^{e}$ siècle, transforma la célébration qui fut désormais "utilisée à des fins symboliques pour exercer et renforcer le pouvoir ${ }^{34}$ ", comme toutes les fêtes civiles et religieuses, certes, mais de manière plus flagrante. Dès le $\mathrm{XVII}^{\mathrm{e}}$ siècle, la Saint Patrick conjuguait importance religieuse et signification séculière. Elle était l'occasion pour protestants et catholiques de toutes les catégories sociales de faire la fête et de boire, souvent plus que de raison. Après une brève interruption à la suite de l'expédition de Jacques II et la guerre qui s'ensuivit entre catholiques et protestants, la commémoration consensuelle reprit de plus belle. À la suite de l'Acte d'Union, la commémoration de Patrick le 17 mars devint une date importante tant pour les deux Églises que pour le château de Dublin. Dans le même temps, la querelle sur l'appartenance religieuse de saint Patrick commençait à faire rage et la deuxième moitié du XIx ${ }^{e}$ siècle verrait s'effriter le consensus autour de la fête annuelle même si l'élite anglo-irlandaise devait continuer d'organiser une parade du drapeau (trooping the colours) à Dublin jusqu’à la première guerre mondiale.

32. Design Research Group, "Incalculable Good, Irreparable Harm : RTÉ, the St Brigid's Cross and the significations of Domesticity ", 2007, [http://designresearchgroup.wordpress.com/2007/05/14/incalculable-good-irreparable-harm-rte-the-st-brigids-cross-and-the-significations-of-domesticity/]. Consulté le 30 septembre 2014.

33. Catholic Herald, UK, 12 February 1937, [http://archive.catholicherald.co.uk/article/12th-february-1937/11/irishletter]. Consulté le 30 septembre 2014.

34. Mike Cronin et Daryl Adair, The Wearing of the Green - A History of St Patrick's Day, Londres et New York, Routledge, 2002, p. XxiI. 
Dans les années 1860 et jusqu'au Peoplés Festival de 1870, les guildes marchandes de Dublin promurent l'idée de la nécessité de profiter de l'occasion pour mettre en avant la dimension purement irlandaise de la célébration. Dès les années 1840, l'Église catholique ${ }^{35}$ s'était singularisée en saisissant l'opportunité pour promouvoir la tempérance par le biais de défilés organisés sur le territoire de toute l'île. Cette tendance, ainsi que la volonté de célébrer tout à la fois l'irlandité et l'appartenance catholique romaine de Patrick allaient trouver sa pleine expression après la Première Guerre mondiale quand la Saint Patrick apparaîtrait de plus en plus nettement liée à la cause de l'indépendance irlandaise. Dès 1903, cependant la Saint Patrick était devenue un jour férié à la suite du vote par le parlement britannique du Bank Holiday (Ireland) Act introduit par le député irlandais James O'Mara. À la suite d'excès de boisson lors des célébrations, ce dernier allait encore s'illustrer, en proposant que les pubs et autres bars soient fermés le 17 mars, une mesure qui fut reprise après l'indépendance en 1922. La fermeture des pubs devait demeurer effective jusqu'en 1970, tandis que la commémoration prenait la forme de services organisés dans toutes les églises, de la participation du gouvernement à la grand-messe dite en gaélique à la Pro-Cathedral de Dublin et d'un défilé militaire dans les rues de la capitale. M. Cronin et D. Adair voient dans la fermeture légale des débits de boisson " un reflet du lien étroit entre l'Église catholique et l'État irlandais ${ }^{36}$ ", mais c'est le célèbre discours prononcé par Eamon de Valera le 17 mars 1943 qui résume le mieux la fusion intime entre le politique et le religieux symbolisé par la fête nationale. Il y parle de l'Irlande dont il rêve, et qu'il cherche à faire advenir, une Irlande dont les habitants mèneraient " la vie que Dieu désire qu'ils vivent " afin d'accomplir la promesse faite par saint Patrick à leurs ancêtres de rendre possible un bonheur identique dans leur pays à celui qu'ils peuvent escompter dans l'autre monde ${ }^{37}$. Le ton est lyrique et le propos induit, d'une certaine manière, la possibilité d'un retour au jardin d'Éden pour une population irlandaise lavée du péché originel grâce aux efforts conjoints de l'Église et de l'État.

Soixante-dix ans plus tard, l'Irlande est méconnaissable. L'alcool coule à flots à la Saint Patrick, et les rêves de 1943 se sont envolés. Le saint national et sa compagne de destinée n'ont pas pour autant été remisés dans les caves du souvenir, bien au contraire. Ils sont en 2014 bien vivants et vigoureux, porteurs de causes adverses mais soutenus avec tout autant d'enthousiasme que lors de leurs incarnations antérieures.

35. Le mouvement de tempérance était également soutenu par les protestants.

36. Ibid., p. 135.

37. Un enregistrement de cet extrait du discours peut être écouté sur Youtube à l'adresse suivante : [https://www. youtube.com/watch?v=-icDyDHI29g]. Site consulté le $1^{\text {er }}$ octobre 2014. 


\section{圈 Patrick et Brigitte aujourd'hui : deux visions rivales du monde}

La place des deux saints dans le paysage culturel et religieux de l'Irlande a considérablement évolué depuis que sont intervenus les changements liés, d'une part, à la crise d'autorité qui a durement affecté l'Église à partir des années 1990 et, d'autre part, à la vague du tout économique qui a balayé l'Irlande du Tigre celtique. L'un et l'autre ont embrassé des causes nouvelles et sont devenus les figures emblématiques d'une Irlande en pleine mutation. La rupture entre l'Église et l'État est ainsi parfaitement symbolisée par le rôle totalement paradoxal que l'on fait aujourd'hui jouer à Patrick. Il est en premier lieu devenu le héros d'une fête internationale qui dépasse largement le monde de la diaspora et n'a plus grandchose à voir avec la célébration essentiellement religieuse et ethnico-nationale, voire tribale, de l'ère De Valera. Sous l'effet de l'américanisation des festivités et de leur marchandisation, saint Patrick s'est fait le héros d'une glorification de l'hédonisme et un chantre de la consommation d'alcool à outrance. Les trèfles en plastique, les chapeaux de Leprechaun à la Walt Disney ${ }^{38}$, la bière verte, les parades spectaculaires, tout va à l'encontre du message que le saint incarnait dans le cadre des commémorations mises en place dans les premières décennies de l'État irlandais. L'objectif est essentiellement économique. À la suite d'une intervention directe du gouvernement, le St Patrick's Festival Committee a été créé en 1995 pour définir une stratégie à visée touristique se fixant pour objectif de faire de Dublin le lieu où il faut être le 17 mars. En 2013, Fáilte Ireland se félicitait d'avoir attiré 140000 visiteurs étrangers et généré un revenu de 121 millions d'euros, tous chiffres en augmentation nette par rapport à la manifestation de 2010, qui avait rassemblé 100000 touristes internationaux pour un gain de 60 millions d'euros ${ }^{39}$. Nous sommes bien loin de la commémoration d'un saint dont la vie était porteuse d'un message religieux pour la nation.

Patrick, apôtre du sécularisme et du consumérisme le jour de la fête nationale d'Irlande, reste pourtant au cœur de la tradition catholique la plus rigide. Il incarne ainsi non seulement la sécularisation spectaculaire de l'État mais, paradoxalement, le maintien de poches de traditionalisme religieux militant, qui se manifeste à la fois sur les sites de pèlerinage de Croagh Patrick et Lough Derg, et dans la société civile en général. Lough Derg demeure ainsi le site par excellence du pèlerinage pénitentiel tel que John Ó’Ríordáin voulait le voir en plein renouveau en 1998, lorsque parut son livre sur la spiritualité catholique irlan-

38. Voir Darby O'Gill and the Little People, Walt Disney, 1959. Ce film est à l'origine des perceptions populaires contemporaines des leprechauns.

39. Fáilte Ireland, «St Patrick's day figures show significant visitor boost », 10 mai 2013, [http://www.failteireland.ie/News-Features/News-Library/St-Patrick-s-Day-figures-show-significant-visitor.aspx]. Consulté le 2 octobre 2014. 
daise. "L'incapacité à comprendre la pénitence est synonyme d'incapacité à comprendre le christianisme irlandais ", écrivait-il alors. Il voyait dans l'acceptation de la mortification du corps "un formidable signe d'espoir et de continuitét ${ }^{0}$ ". Aujourd'hui, l'aspect de continuité et d'intemporalité est particulièrement mis en avant. La survie du pèlerinage de Lough Derg est décrite comme "un mystère, un miracle, un besoin, un défi ${ }^{41}$ ". Le pénitent s'y purifie " des couches de culpabilité, de souffrance et de peur » dans " un lieu reculé, vierge de tout contact avec le monde moderne ${ }^{42}$ ", un espace " intemporel, sacré et baigné des prières de nos ancêtres $^{43}$ ". Le message est comparable à Croagh Patrick, où l'on accueille les fidèles " dont la foi a été ébranlée par la montée du matérialisme et les défis permanents du consumérisme ", ceux, aussi, qui souhaitent se joindre à une expérience fondamentalement collective " qui se dresse contre l'individualisme omniprésent ${ }^{44} »$.

Certes, Lough Derg est aussi un projet économique réussi, qui a reçu en 2004 l'Excellence Ireland Foundation Mark for Effective Management et a su se mettre en quête d'un nouveau public moins traditionaliste par voie d'affichage sur les bus dublinois et le Dart, au cours de sa campagne publicitaire baptisée Soul Survival Campaign ${ }^{45}$. Mais si la réalité contemporaine n'est pas absente des sites de pèlerinage traditionnels, il est indiscutable que les pèlerins s'y rendent encore souvent dans un esprit de pénitence comparable à celui de leurs prédécesseurs. Leur nombre est néanmoins en baisse, même si le site a accueilli encore 18000 pèlerins en $2013^{46}$.

En dehors des centres anciens de dévotion, Patrick incarne aussi pour beaucoup une Église conservatrice caractérisée par son attachement profond à l'institution. Notons, à titre d'exemple, la création en 2013 de la Confraternité irlandaise du clergé catholique (Irish Confraternity of Catholic Clergy), placée essentiellement sous la protection de Notre-Dame de Knock et de saint Patrick. Cette structure se fixe pour objet premier d'agir pour la préservation de la tradition catholique en manifestant sa fidélité absolue au magistère de l'Église, pouvoir d'enseignement doctrinal « institué autoritairement par le Saint-Père et les évêques en communion avec lui ${ }^{47}$ ". Un tel engagement se situe au cœur des préoccupations de cercles catholiques héritiers des enseignements du $\mathrm{xIx}^{\mathrm{e}}$ siècle. Il se démarque nettement de groupes progressistes comme l'Association des prêtres catholiques (Association

\footnotetext{
40. John Ó’Ríordáin, Irish Catholic Spirituality Celtic and Roman, Dublin, Columba Press, 1998, p. 126-127.

41. Eileen Good, op. cit., p. 1.

42. Ibid., p. 69.

43. Ibid., p. 80.

44. Harry Hughes, op. cit., p. 1, préface de l'archevêque Michael Neary.

45. «Lough Derg Revival - Pilgrims' Progress », Irish Independent, 15 juin 2004.

46. "Call to Pilgrims to support the ancient Lough Derg Pilgrimage ", The Irish Times, 27 août 2014.

47. [http://confraternity.ie]. Site consulté le 3 octobre 2014.
} 
of Catholic Priests) qui, pour respecter le credo à la lettre, se fait souvent critique du gouvernement de l'Église ${ }^{48}$.

Les franges conservatrices savent également aujourd'hui avoir recours aux moyens modernes de communication pour véhiculer leurs convictions, ainsi que l'atteste la diffusion du documentaire intitulé St Patrick Apostle of Ireland (2004). La teneur des propos qui y sont tenus n'est pas sans évoquer les Vies du XIXe siècle signalées plus haut, en raison de l'absence de recul critique manifeste vis-à-vis des interpolations médiévales ${ }^{49}$. Certes, le bannissement des démons à Croagh Patrick reçoit un traitement psychologique qui rend l'épisode métaphorique : ce sont ses démons intérieurs que Patrick a chassés au cours de son expédition sur la montagne sacrée; mais l'historicité de sa visite en ce lieu n'est pas plus remise en cause que l'envergure nationale de la mission patricienne. Ce documentaire contraste singulièrement avec le film de télévision, significativement nommé St Patrick, the Irish Legend (2000), qui évoque les mêmes histoires miraculeuses, mais les présente comme totalement légendaires ${ }^{50}$. Le documentaire n'est toutefois pas totalement déconnecté des réalités contemporaines puisque, à l'inverse d'auteurs comme Bury, il fait de Patrick un apôtre du christianisme celtique, à la mode depuis une vingtaine d'années, et il lie son culte contemporain au respect de la planète, une orientation forte chez les rénovateurs du message chrétien.

Si Brigitte, elle aussi, continue à être vénérée de manière traditionnelle à Faughart et même, dans une bien moindre mesure, à Lough Derg, elle connaît, depuis les années 1990 une véritable renaissance. Acte symbolique s'il en est, en 1992, l'ordre de Sainte-Brigitte établi à Tullow au début du XIX ${ }^{e}$ siècle a fondé une petite communauté à Kildare, un retour assumé aux origines : "Nous, sœurs de Sainte-Brigitte, sommes venues vivre à Kildare en août 1992, pour retrouver le lien avec nos racines celtiques et nous réapproprier Brigitte d'une nouvelle manière à l'aube du nouveau millénaire ${ }^{51}$. » En 1993, conjointement avec l'ONG Action from Ireland (AFRI), les sœurs ont organisé un congrès international sur le thème suivant "Brigid: Prophetess, Earthwoman, Peacemaker». Le feu de Brigitte a été rallumé à l'ouverture de la manifestation; il est entretenu en permanence depuis cette date. L'objectif des deux initiatrices du projet était de promouvoir la spiritualité celtique dans un cadre totalement œecuménique qui leur permette de rester attachées à leurs racines chrétiennes tout en redonnant vie à la déesse païenne, incarnation de la terre d'Irlande. Elles collaborent ainsi avec des

48. Pour s'en convaincre, consulter le site de l'association à l'adresse suivante: [http://www.associationofcatholicpriests. ie]. Consulté le 3 octobre 2014.

49. DVD St Patrick Apostle of Ireland, 2004.

50. St Patrick: the Irish Legend, 2000, film entier disponible sur Youtube à l'adresse suivante : [https://wwwwyoutube. com/watch? $v=n d U f g H o p]$. Visionné à cette adresse le 9 septembre 2014.

51. « Kildare since 1992 », Site web du centre de Solas Bhríde [http://solasbhride.ie/85-2/]. Site consulté le 3 octobre 2014. 
protestants et des néo-païens et s'en expliquent de la manière suivante : "Nous travaillons avec ceux qui sont en quête d'une spiritualité qui soit pertinente dans le monde d'aujourd'hui ${ }^{52}$. " Elles se sentent investies de la mission d'" utiliser la sagesse ancienne pour les temps nouveaux " et leur programme comprend, entre autres, une action déterminée en faveur de "la survie écologique de la planète ", de la justice sociale et d'" un pouvoir accru des femmes ». Dans leur ouvrage sur la Saint Patrick, M. Cronin et D. Adair notent que «l'histoire de l'anniversaire patricien doit être compris dans le contexte d'une domination masculine et d'une marginalisation des femmes ${ }^{53}$ ". Brigitte aujourd'hui incarne la féminisation du monde qui, associée à " la progressivité réinvestissant les archaïsmes ${ }^{54}$ ", produit ce que Michel Maffesoli nomme « l'invagination du sens $s^{55}$ ».

Depuis 1993, le Festival annuel de Sainte Brigitte, la Féile Bhride, est fixé début février et il dure une semaine. Il est centré autour de l'idée de " culte créatif " qui met les artistes au cœur de la célébration, rappel s'il en est que la déesse païenne, comme la sainte, protégeaient les poètes et les artisans. Les artistes sont symboliquement appelés à joindre leurs efforts à ceux de Brigitte pour générer une énergie créatrice susceptible de donner naissance à un nouvel avenir. Il était essentiel pour les organisateurs de s'inscrire dans la tradition d'Imbolc, la fête préchrétienne du $1^{\text {er }}$ février, au seuil du printemps annonciateur de la renaissance annuelle de la nature. Le retour au calendrier cyclique des Celtes fait écho au réinvestissement de l'espace archaïque. Il est intéressant à ce titre de remarquer que, si l'événement traditionnel de l'été a été maintenu à Faughart, un nouveau pèlerinage y a été créé par Dolores Whelan en 2013 qui relie, toujours en juillet, Faughart à Kildare.

Le retour de Brigitte rencontre un grand succès, qui dépasse largement les pèlerinages et le domaine religieux. Nous ne nous attarderons pas ici sur Le Jardin de Brigitte (Brigit's Garden), ouvert en 2004 dans les environs de Galway, et dont la fondatrice, Jenny Beale voulait faire un lieu " calme et magnifique susceptible de nourrir une spiritualité adaptée au $\mathrm{XXI}^{\mathrm{e}}$ siècle " tout en célébrant "la nature et le cycle de la vie ${ }^{56}$ ". Il est en effet plus pertinent de revenir à la question de la dimension nationale de la sainte, comme nous l'avons fait pour Patrick. Un faisceau d'indications semble nous permettre de suggérer que non seulement Brigitte est toujours une sainte nationale mais que son envergure a crû. Signe s'il en est du soutien politique aux évolutions relatées plus haut, la présidente de la République

52. Ibid., "Our history », [http://solasbhride.ie/our-history/]. Site consulté le 3 octobre 2014.

53. Mike Cronin et Daryl Adair, op. cit., p. 249.

54. Michel Maffesoli, Matrimonium - Petit traité d'écosophie, Paris, CNRS édition, 2010, p. 11.

55. Ibid., p. 7.

56. "The Story of Brigit's Garden by its Founder, Jenny Beale " : [http://www.brigitsgarden.ielabout-us/the-story-ofBrigit-s-garden]. Noter également une interview très intéressante de Jenny Beale sur Youtube : [https://www. youtube.com/watch? $v=A W Z D Y B p F c d 4$ ]. Les deux sites ont été consultés le 3 octobre 2014. 
d'Irlande Mary McAleese a, le $1^{\text {er }}$ février 2006, dévoilé, à Kildare, le monument à la flamme de Brigitte et allumé un second feu de Brigitte issu de celui entretenu par les religieuses à leur centre de Solas Bhríde. De même, un examen de la vogue étonnante de la sainte outre Atlantique et dans toute la diaspora convainc l'observateur qu'elle incarne l'Irlande dont rêvait Mary Robinson dans son discours inaugural en 1990 : une Irlande internationale qui englobe les 70 millions de descendants des émigrants irlandais; une Irlande où les femmes trouvent leur juste place; une Irlande, enfin, où l'on célèbre la nation par les arts et la justice sociale $^{57}$.

En 1995, les Sœurs de Sainte Brigitte ont été appelées à porter la flamme de Kildare au Royal Albert Hall de Londres, où elles ont ouvert un colloque sur "Ce que veulent les femmes ». À cette occasion, elles ont lancé un concert rock avec « un rituel incorporant la flamme et le tressage de croix de sainte Brigitte ». Ce symposium s'est tenu en vue de la préparation d'une conférence de femmes à Pékin, où la chanteuse internationale Nóirín Ní Riain a apporté la flamme, qui a brûlé pendant toute la durée de la manifestation. Depuis, elle a été portée à La Haye, à Iona, en Australie, en Nouvelle-Zélande, aux États-Unis et bien d'autres lieux pour éclairer diverses conférences sur la paix.

Le nombre impressionnant de publications internationales de ces dix ou quinze dernières années sur le sujet de Brigitte ainsi que la dimension internationale des commémorations donnent à penser que la sainte répond aux besoins spirituels et aux idéaux humanitaires ou socio-politiques de beaucoup. Ces célébrations ne sont d'ailleurs pas systématiquement collectives, comme l'indique, par exemple le fait que Brigitte est devenue une héroïne de l'Internet, où se retrouvent les adeptes de toutes sortes de spiritualités et religions établies pour célébrer la sainte et/ou la déesse le $1^{\text {er }}$ février de chaque année. Un festival international de poésie a ainsi été lancé en 2006, qui a rencontré un grand succès et est aujourd'hui présenté comme " une tradition de l'Internet ». Le principe en est simple : chacun est sollicité pour poster un poème afin de contribuer à la célébration mondiale de Brigitte.

À visée collective, de telles commémorations virtuelles ont de facto une forte dimension privée puisque chaque participant est seul devant son ordinateur. Il est dès lors permis de poser l'hypothèse que les commémorations contemporaines de Brigitte constituent un exemple de ce que F.R. Ankersmit analyse comme la privatisation du passé qui a, selon lui, pour effet une privatisation des commémorations ${ }^{58}$.

57. Mary Robinson, "Inauguration Speech », 1990, éd Richard Aldous, Great Irish Speeches, London, Quercus, 2007, p. 169-170.

58. F. R Ankersmit, "Commemoration and National Identity ", Textos De História, Vol. 10 1-2, 2002, p. $23-29$. Accessible en ligne : [http://fr.scribd.com/doc/177783214/Ankersmit-Commemoration-and-National-Identity]. Consulté le 3 octobre 2014. 
Dans le cas de Brigitte, cette privatisation fait écho à la fragmentation des croyances religieuses et à la privatisation du religieux en général. Gérard Namer suggère que les commémorations publiques traditionnelles contrôlées par une autorité centrale visaient à légitimer l'autorité de leurs organisateurs ${ }^{59}$. Il est permis de suggérer que l'inverse est vrai également et que les commémorations privées ou réservées à de petits groupes permettent de légitimer de petites communautés marginales en rupture par rapport à l'autorité et l'institution, si nous parlons de l'Église catholique.

Qu'il nous suffise, avant de conclure, de citer deux exemples. Le premier concerne la Catholic Faith Community, basée à Calgary (Canada). Ses membres se réclament de Jésus Christ et Ste Brigitte, mais l'organisation appartient à un mouvement appelé Roman Catholic Womenpriests qui, comme son nom l'indique, est favorable à l'ordination des femmes. Elle est dirigée par une femme prêtre, qui a été ordonnée par une femme évêque. Leur objectif est de promouvoir le féminisme catholique et une compréhension œecuménique de la tradition sacramentelle catholique ${ }^{60}$. L'autre exemple est celui de la communauté virtuelle globale baptisée l'abbaye des Arts, de tradition catholique, qui vénère Ste Brigitte avant tout, mais souhaite être à l'origine d'une "révolution monastique " visant à une prise de conscience écologique par le biais de la pratique artistique ${ }^{61}$.

Nous le voyons, Brigitte la multiforme incarne aujourd'hui notamment l'idée du renouveau et de la dissidence par rapport à l'institution. Elle est l'expression de l'éclatement de la cohérence religieuse catholique d'antan, ainsi que du succès du relativisme et du syncrétisme. Elle est toujours une sainte nationale irlandaise, mais dans une acception nouvelle, puisqu'elle symbolise aussi l'Irlande de la diaspora et, plus généralement, une Irlande ouverte sur l'international, une Irlandemonde. Patrick reste associé aux rigueurs d'un catholicisme romain très conservateur, totalement inféodé à Rome. Mais le saint national de la fête du 17 mars s'est encanaillé, il est marqué au sceau du consumérisme, de l'excès et, pour tout dire, de la sécularisation et de la modernisation de l'État irlandais. Leurs commémorations, elles aussi, s'opposent et se contredisent. Il ressort de leur analyse que Patrick est holiste et que, dans un contexte de crise d'autorité, il légitime les pouvoirs établis. Brigitte en revanche est individualiste et justifie la position des rebelles à l'autorité. Tous deux cependant sont les fers de lance de visions du monde et de choix d'avenir. Des débats et tensions qu'ils génèrent sortira immanquablement la voie négociée vers un futur acceptable par tous. Qui sait alors ce que sera la destinée des deux saints nationaux?

59. Gérard Namer, Mémoire et société, Paris, Méridiens Klincksieck, 1987, p. 205.

60. Pour plus d'information, consulter le site web de cette organisation à l'adresse suivante: [http://saintbrigids.org]. Site consulté le 3 octobre 2014.

61. Ils sont basés à Galway, mais ont également une adresse à Houston, Texas. Pour plus d'information, consulter le site web de cette organisation à l'adresse suivante : [http://abbeyofthearts.com]. Site consulté le 3 octobre 2014. 